\title{
Effect of Lymphocyte Immunotherapy on Pro-Inflammatory Th17 Related Cytokine Levels in Women with Recurrent Spontaneous Abortion
}

\section{A R T I C L E I N F O}

\section{Article Type}

Original research

\section{Authors}

Zare A. ${ }^{*} P h D$,

Saremi A. ${ }^{1} M D$,

Salehian P. ${ }^{1} M D$

Roomandeh N. ${ }^{2} M S c$,

Naderi M. ${ }^{1} B S C$,

Lashgari P. ${ }^{1} B S c$,

Arasteh J. ${ }^{3} P h D$,

Kokhaei P. ${ }^{2} P h D$

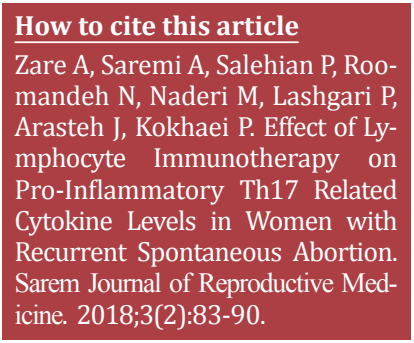

*Sarem Cell Research Center, Sarem Women's Hospital, Tehran, Iran ${ }^{1}$ Sarem Cell Research Center, Sarem Women's Hospital, Tehran, Iran ${ }^{2}$ Cancer Research Center and Department of Immunology, Faculty of Medicine, Semnan University of Medical Sciences, Semnan, Iran ${ }^{3}$ Department of Biology, Faculty of Basic Sciences, Central Tehran Branch, Islamic Azad University, Tehran, Iran

\section{Correspondence}

Address: Sarem Women's Hospital, End of Phase 3, Ekbatan Town, Tehran, Iran

Phone: +98 (21) 44670888

Fax: +98 (21) 44670885

ahadzr@gmail.com

\section{Article History}

Received: December 23, 2017

Accepted: April 09, 2018

ePublished: June 15, 2018

\section{A B S T R A C T}

Aims Alterations of Th17 cells during pregnancy have a crucial role in maintenance of fetus and the high level of these cells has been observed in women with recurrent spontaneous abortion. Immunotherapy with paternal lymphocytes is one of the effective therapeutic methods for this complication. The aim of this study was to evaluate the alteration in Th17 cytokine levels in patients with recurrent abortion after lymphocyte immunotherapy.

Materials \& Methods In this semi-experimental study, 30 patients referred to Sarem hospital with a history of at least three recurrent abortions in 2013 were evaluated. The levels of IL17 and IL-21 in patient serum and supernatant of cultured peripheral blood mononuclear cells were assayed before and after this treatment by using ELISA. Statistical analysis was performed with Wilcoxon test by SPSS 22.

Findings The levels of IL-17and IL-21 in patient serum after immunotherapy were significantly decreased $(\mathrm{p}<0.05)$. Supernatant of cultured peripheral blood mononuclear cells after immunotherapy showed a low level of IL-21 ( $<<0.04)$, while this change was not significant for interleukin $17(\mathrm{p}=0.13)$.

Conclusion Lymphocyte immunotherapy reduces the level of pro-inflammatory cytokines of Th17 cells in women with recurrent spontaneous abortion.

Keywords Immunotherapy; Habitual Abortion; Thıv cells

\section{I T A T I O N L I N K S}

[1] Why isn't the fetus ... [2] The Th1/Th2/Th17/Treg paradigm induced by stachydrine hydrochloride reduces ... [3] Reproductive ... [4] An update in recurrent spontaneous ... [5] Recurrent miscarriage: Causes, evaluation and ... [6] Abnormal embryonic karyotype is the most frequent cause of recurrent ... [7] Recurrent pregnancy loss: A disease of inflammation ... [8] Inflammation and pregnancy: The role of the immune system at the implantation ... [9] Bidirectional cytokine interactions in the maternal-fetal relationship: Is successful ... [10] Aberrant cytokine production by peripheral blood mononuclear cells in ... [11] Th1/Th2/Th17 and regulatory T-cell paradigm in ... [12] TH17 cells in development: An updated view of their molecular ... [13] The biological functions of T helper 17 cell effector cytokines ... [14] Kuchroo VK. IL-17 and Th17 ... [15] The impact of Th17 cells on transplant rejection and the induction of ... [16] Inflammation and the balance of Treg and Th17 cells in transplant ... [17] Anti-type V collagen lymphocytes that express IL-17 and IL-23 induce ... [18] How do regulatory T ... [19] The balance of the immune system between T cells and NK cells in ... [20] Accumulation of IL-17-positive cells in decidua of inevitable ... [21] Th17 and regulatory T cells in women with ... [22] TH17 cells in human recurrent pregnancy loss and ... [23] Induction of MLR-Bf and protection of fetal ... [24] Lymphocyte immunotherapy and its probable mechanism ... [25] Possible mechanisms of immunotherapy for maintaining pregnancy ... [26] Association of human leucocyte antigen sharing with ... [27] Diagnosis and treatment of recurrent miscarriage ... [28] The influence of paternal lymphocyte ... [29] Alteration of Th17 and Treg cells in patients with unexplained ... [30] Correlation between serum zinc levels and successful immunotherapy ... [31] Cytokines in recurrent pregnancy ... [32] Characteristics of immune cell changes ... [33] Natural killer cells promote immune ... [34] Alteration of TH1 and TH2 cells by intracellular cytokine ... [35] The deregulation of regulatory T cells on interleukin-17-producing T ... [36] Proportional change of CD4+CD25 + regulatory T cells in ... [37] An imbalance in interleukin-17producing $\mathrm{T}$ and ... [38] Intravenous immunoglobulin G modulates peripheral ... [39] The basis and value of currently used ... [40] Effect of leukocyte therapy ... [41] Comprehensive analysis of peripheral blood ... [42] Study on the relationship between Th17 cells ... [43] Genetic variations in the interleukin-21 ... [44] Th17 cells and related cytokines in unexplained ... 


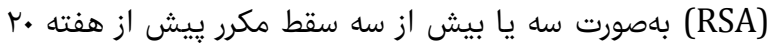

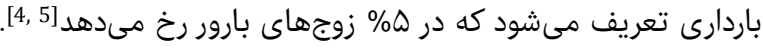

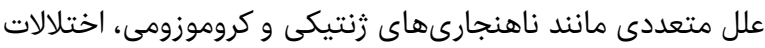

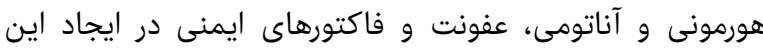

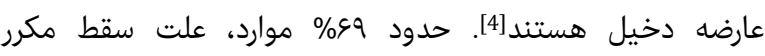

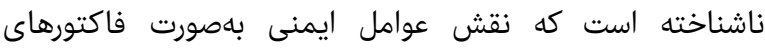
خودايمن و آلوايميون در اين زمينه بيش اعنا ازئ علل ديكر مطرح

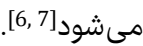

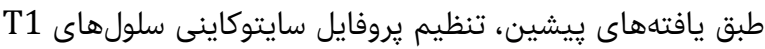

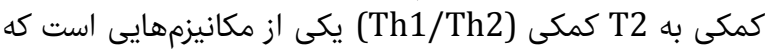

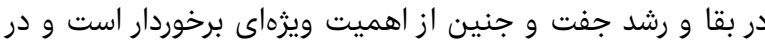

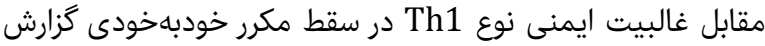

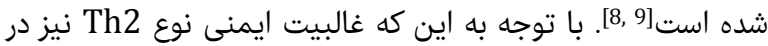

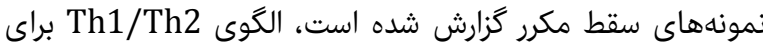

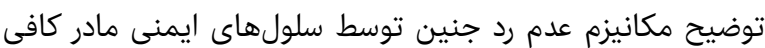

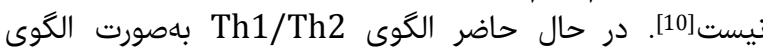
سلولهاى Th1/Th2/Th17 و سلولهاى T إن تنظيمى كسترش

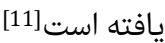

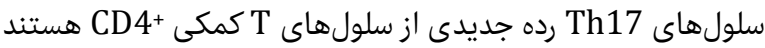

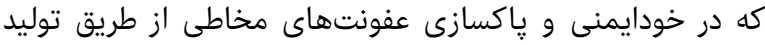

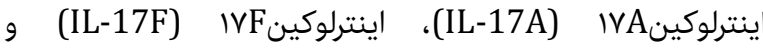

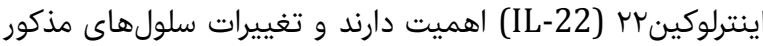

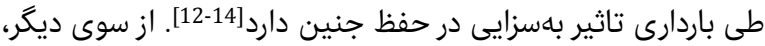

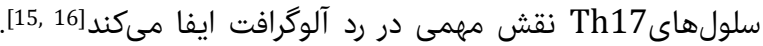

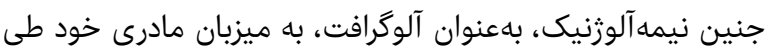

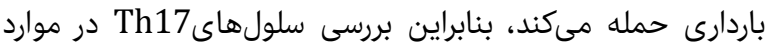

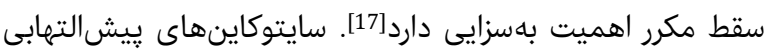
مانند IL-17 و IL-21 كه توسط سلولهاي

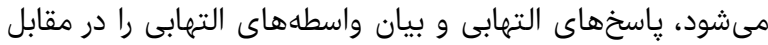

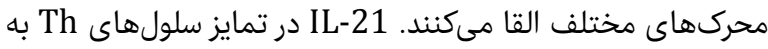

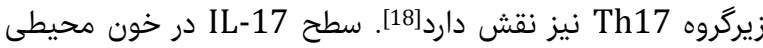

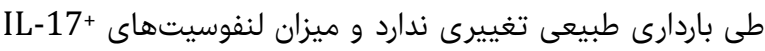

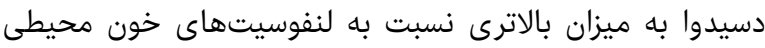

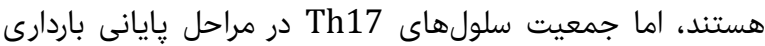

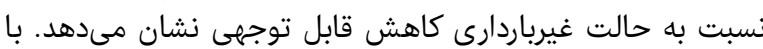

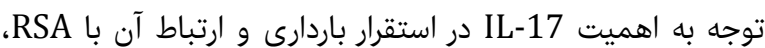

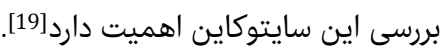

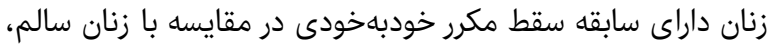

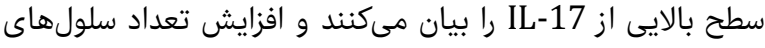

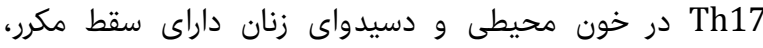

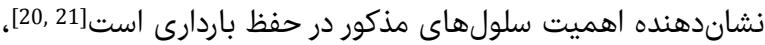

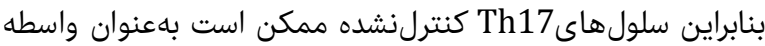

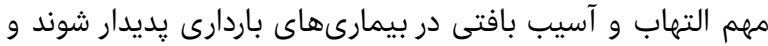

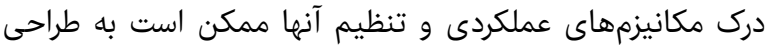

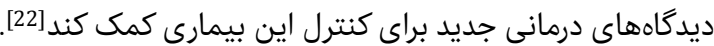

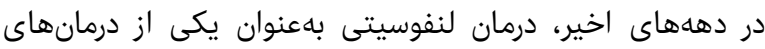

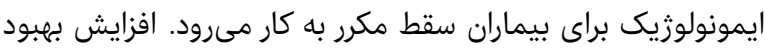

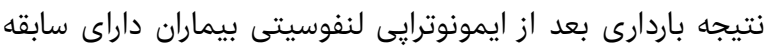

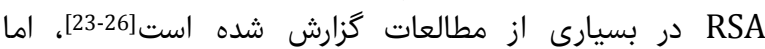

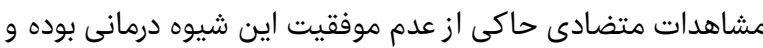

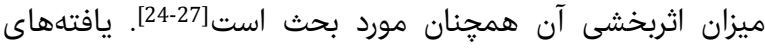

اثر درمان لنفوسيتى بر سطح سايتوكاينهاى ييشالتهابى سلولهاى Th17 در زنان مبتلا به به بله

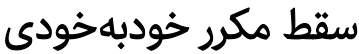

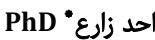
مركز تحقيقات سلولى صارم، بيمارستان صارم، تهران، ايران

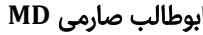
مركز تحقيقات سلولى صارم، بيمارستان صارم، مارم، تهران، ايران MD بيروز صالحيان مركز تحقيقات سلولى صارم، بيمارستان صارم، تهران، ايران

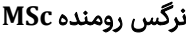

مركز تحقيقات سرطان و وركن سخش ايمنىشناسى، دانشكده يزشكى، دانشكاه علوم

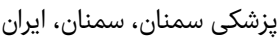

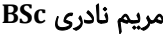
مركز تحقيقات سلولى صارم، بيمارستان صارم، تهران، ايران

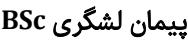
مركز تحقيقات سلولى صارم، بيمارستان صارم، تهران، ايران PhD Pواد آراسته

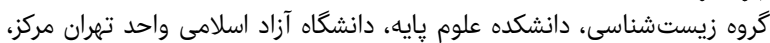

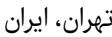

PhD برويز كوخايى ايران ترطان مركز تحقيقات سرطان و بخش ايمنىشناسى، دانشكده يزشكى، دانشكاه علوم يزشكى سمنان، سمنان، ايران

جكيده

اهداف: تغييرات سلولهاى Th17 طى باردارى نقش مهمى در حفظ جنين دارد

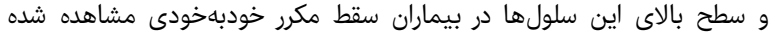

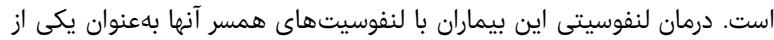

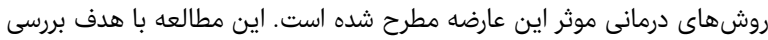

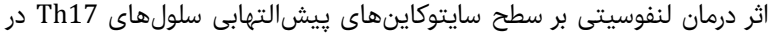

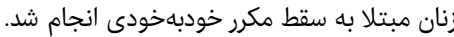

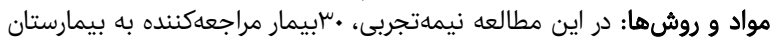

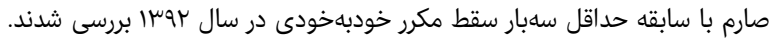

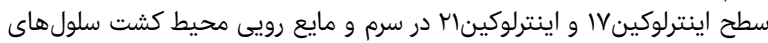

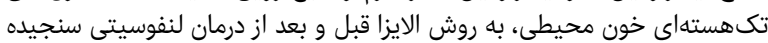

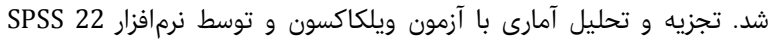

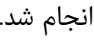

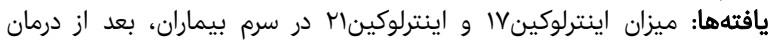

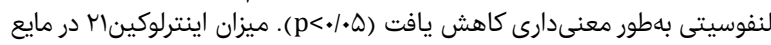

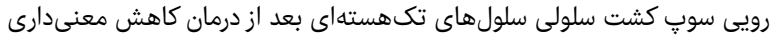

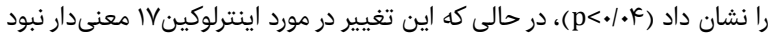

(p=•//

نتيجهيرى: درمان لنفوسيتى سطح سايتوكاينهاى يُشيشالتهابى سلولهاى

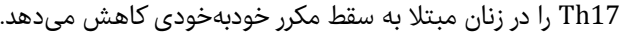

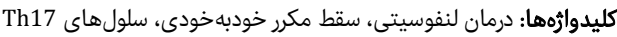

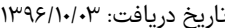

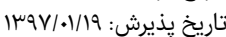

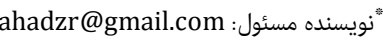

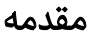

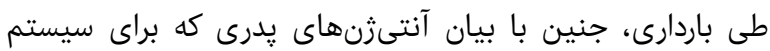

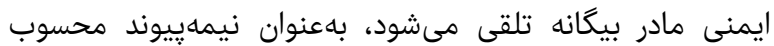

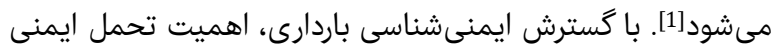

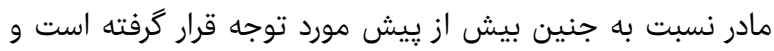

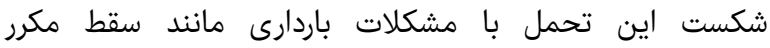

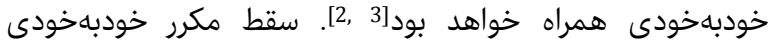




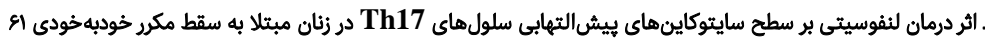

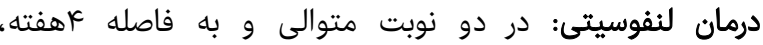

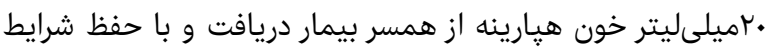

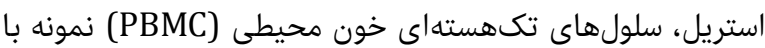

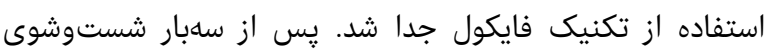

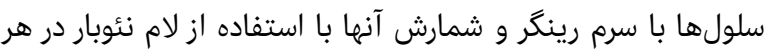

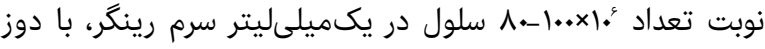

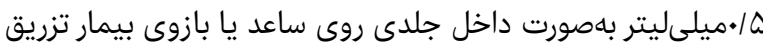

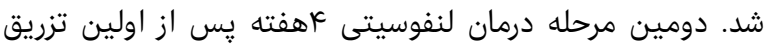

صورت كرفت[30]

سنجش ميزان IL-17 و IL-21 در سرم و سوب كشت سلوكائى براى سنجش سطح سايتوكاينهاى IL-17 و

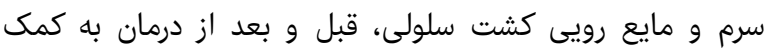

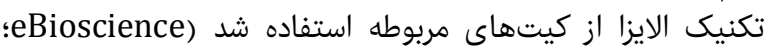

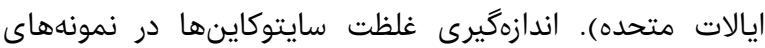

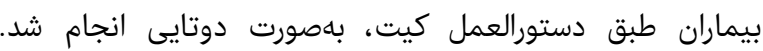

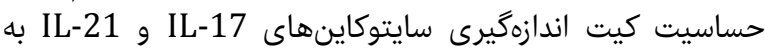

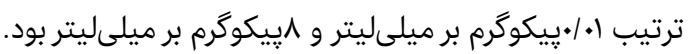
بهمنظور تجزيه و تحليل دادهها از نرمافزار

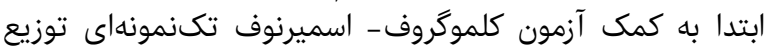

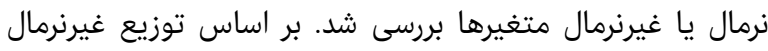

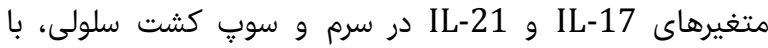

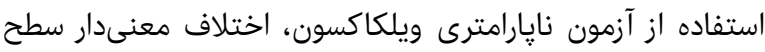

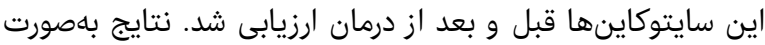

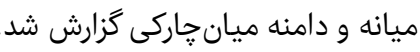

يافتهها

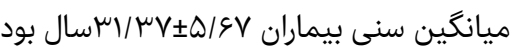

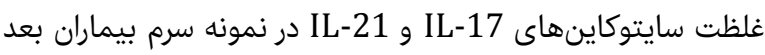

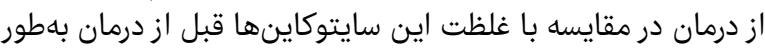

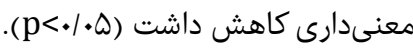

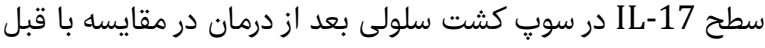

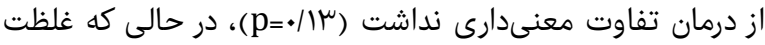
IL-21

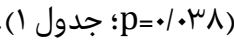

جدول () سطح سايتوكاينها در سرم و مايع رويى كشت سلولى قبل و بعد از

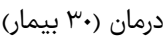

\begin{tabular}{|c|c|c|}
\hline مايع رويى & سرم & سايتوكاين \\
\hline 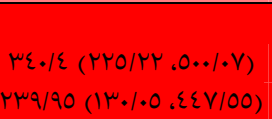 & 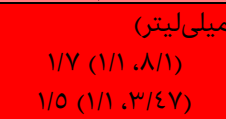 & 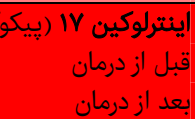 \\
\hline 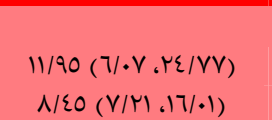 & 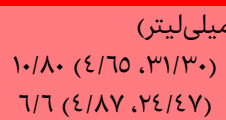 & بعل اينترلوكين از دران إييك \\
\hline
\end{tabular}

بحث

طى باردارى، سايتوكاينهايى كه توسط سلولهاى مادر و جنين

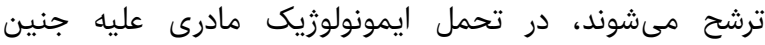

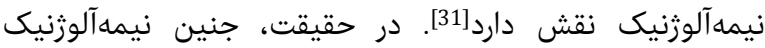

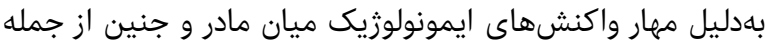

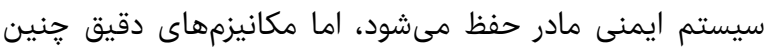

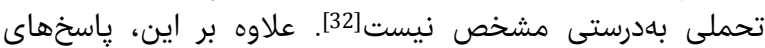

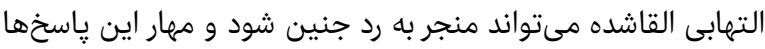

قبلى حاكى از غالبيت سلولهاى Th2 پِس از درمان لنفوسيتى

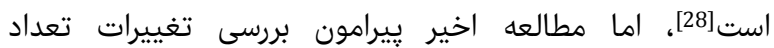

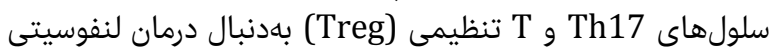

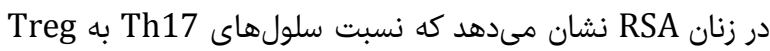

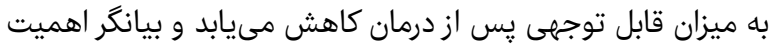
درمان لنفوسيتى براى تغيير عملكرد سلولهاي إن دراي

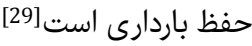
مطالعه حاضر با هدف براد براستى اثر درمان لنفوسيتى بر سطح

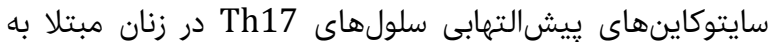
سقط مكرر خودبهخودى انجام شد.

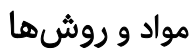

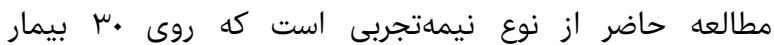

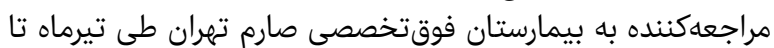

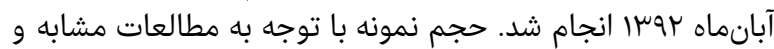

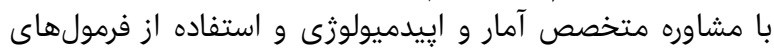

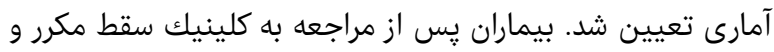

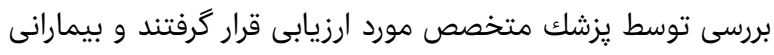

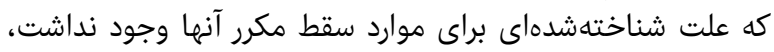

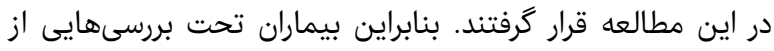

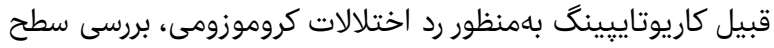

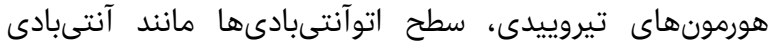

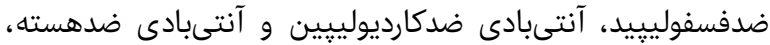

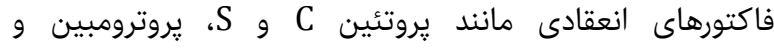

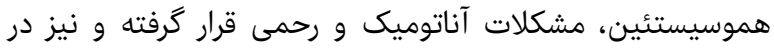

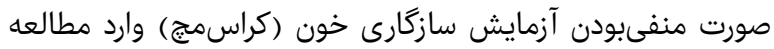

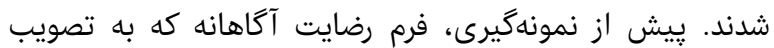

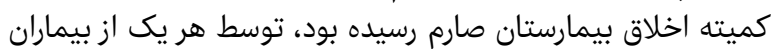

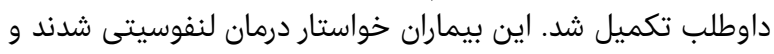

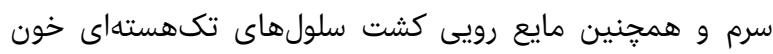
محيطى آنها قبل و بعد از درمان لنفوسيتى از نظر سطح اينترلوكين

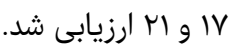
جداسازى سرم: حدود هميلىليتر خون بيماران قبل و بعد از درمان،

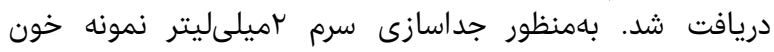

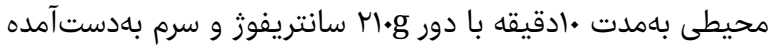

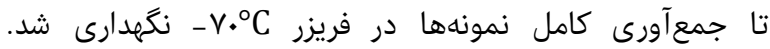

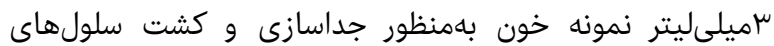

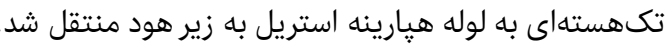

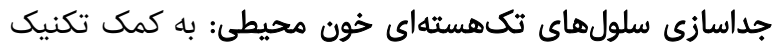

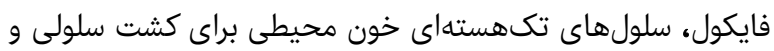

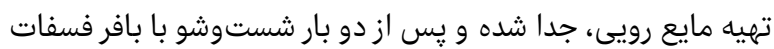

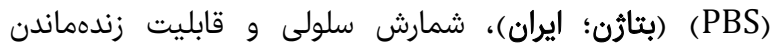

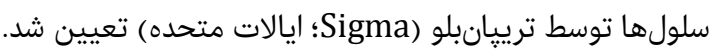

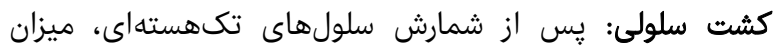

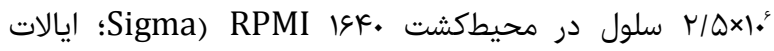

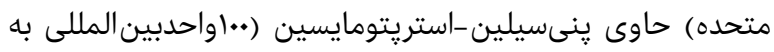

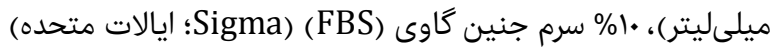

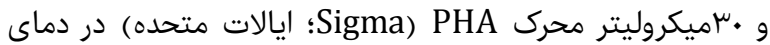

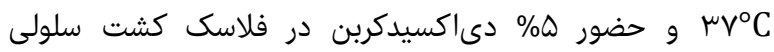

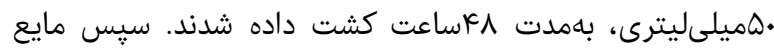

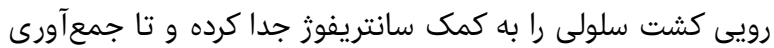

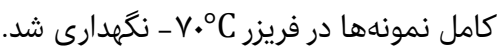


نشانكر كاهش عملكرد التهابى سلول Th17 باشد (به ترتيب

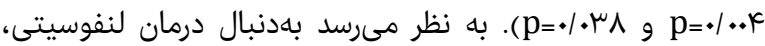

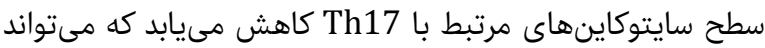

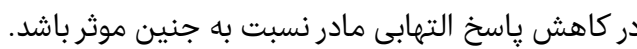

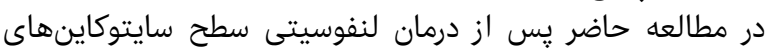

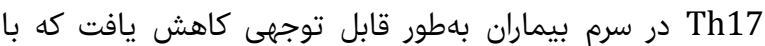

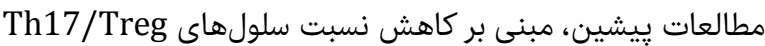

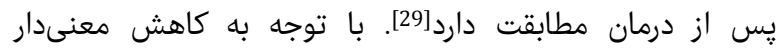

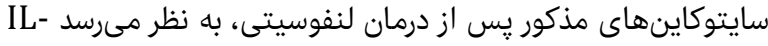

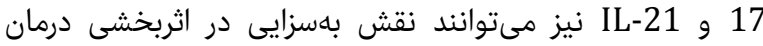

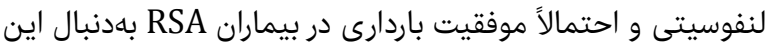

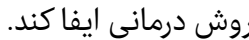

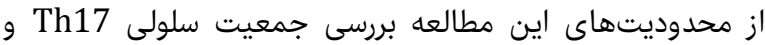

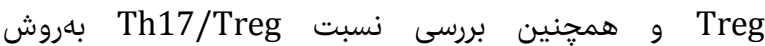

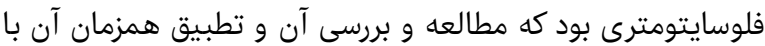

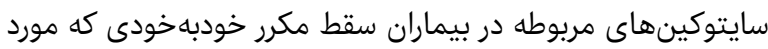
درمان لنفوسيتى قرار مى گيرند، يِيشنهاد مى شودا.

\section{نتيجه ليرى}

درمان لنفوسيتى موجب كاهش سطح سايتوكاينهاى ييشالتهابى زئل

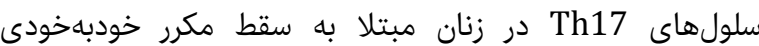

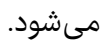

تشكر و قدردانى: از همكاران ديارتمان سقط مكرر و گروه

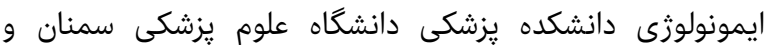

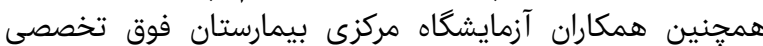

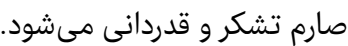

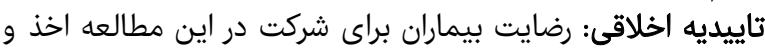

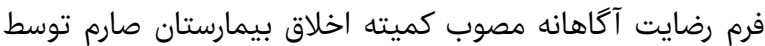

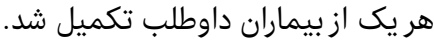
تعارض منافع: موردى توسط نويسندأنان كزارش نشار نشده است.

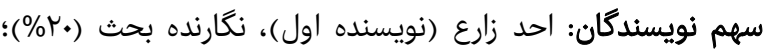

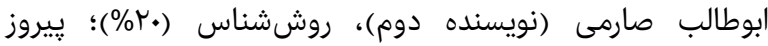

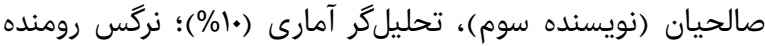

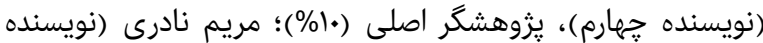

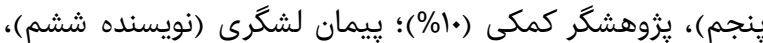

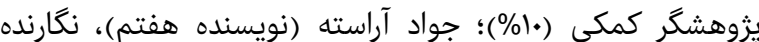
مقدمه (•(\%)؛ يرويز كوخايى (نويسنده هشتم)، نكارنده مقدمه (رئه

منابع مالى: مركز تحقيقات سلولى صارم و دانشگاه علوم يزشكى سمنان منابع مالى اين يزوهش رات را تامين نمودهاند.

منابع

1- Erlebacher A. Why isn't the fetus rejected?. Curr Opin Immunol. 2001;13(5):590-3

2- Li X, Wang B, Li Y, Wang L, Zhao X, Zhou X, et al. The Th1/Th2/Th17/Treg paradigm induced by stachydrine hydrochloride reduces uterine bleeding in RU486induced abortion mice. J Ethnopharmacol. 2013;145(1):241-53.

3- Christiansen OB. Reproductive immunology. Mol Immunol. 2013;55(1):8-15.

4- Pandey MK, Rani R, Agrawal S. An update in recurrent

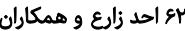

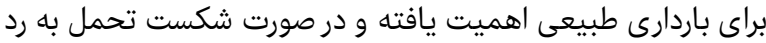

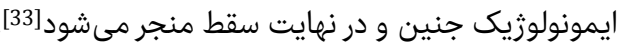

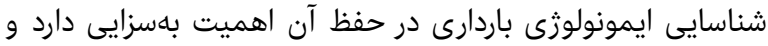

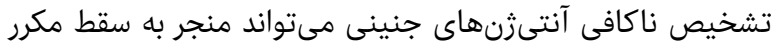

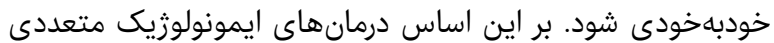

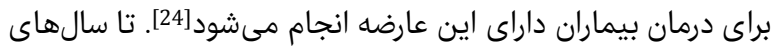

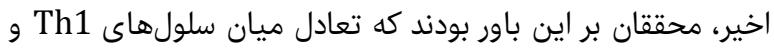

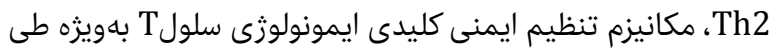

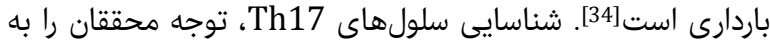

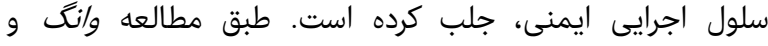

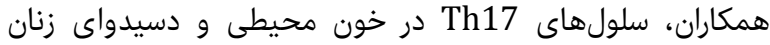
Th17 افزايش و ميزان سايتوكاين RSA

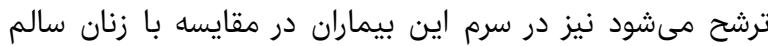

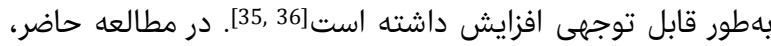

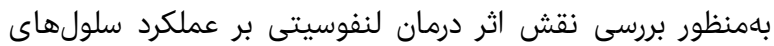

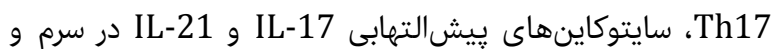

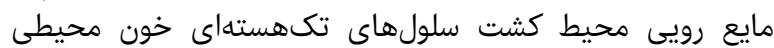
تحريك شده با ميتوزن (PHA) در بيماران داراى سابقه سقط مكاى مكرر قبل و بعد ازدرمان سنجيده شد. يافتههاى اخير تغيير در ازدران سنبده شند سلولهاى اجرايى ايمنى

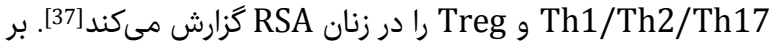

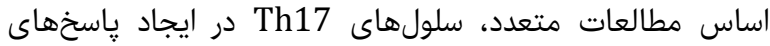

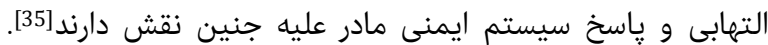

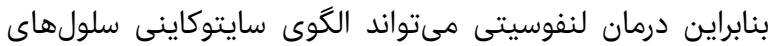

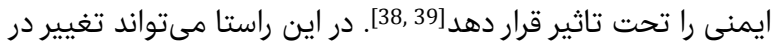

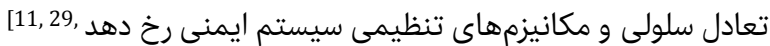

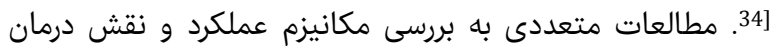

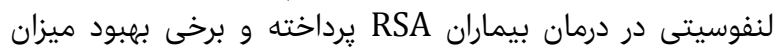

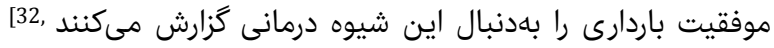

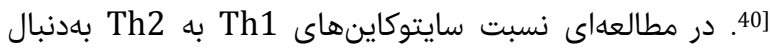

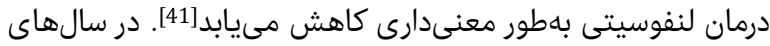

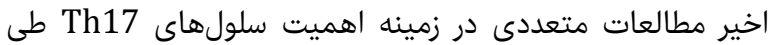

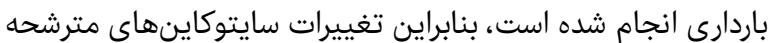

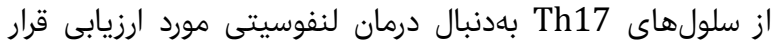

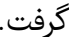

در مطالعهاى نسبت سلولهاى Th17 به سلولهاى Treg يس از

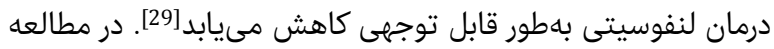

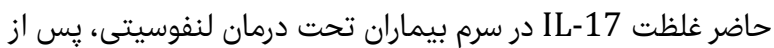

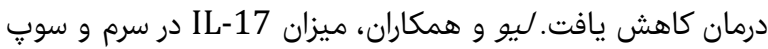

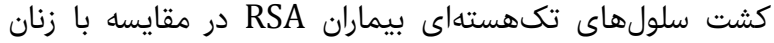

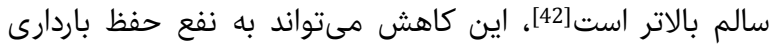

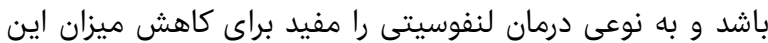
سايتوكاين معرفى كند.

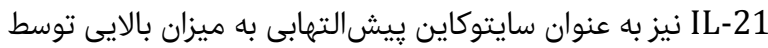

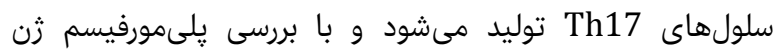

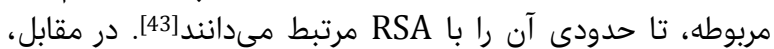

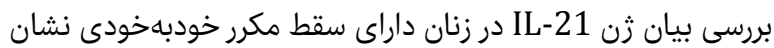

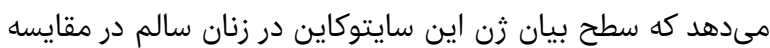

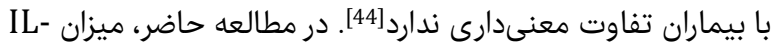

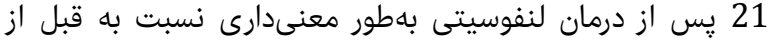
درمان در سرم و مايع رويى كشت سلولى بلى كاهش يافت كه مى نواند 
ني ير سطح سايتوكاينهاى بيشالتهابى سلولهاى Th17 در زنان مبتلا به سقط مكرر خودبهخودى سع for women with recurrent spontaneous abortion. Int Immunopharmacol. 2004;4(2):289-98.

24- Pandey MK, Thakur S, Agrawal S. Lymphocyte immunotherapy and its probable mechanism in the maintenance of pregnancy in women with recurrent spontaneous abortion. Arch Gynecol Obstet. 2004;269(3):161-72.

25- Ito K, Tanaka T, Tsutsumi N, Obata F, Kashiwagi N. Possible mechanisms of immunotherapy for maintaining pregnancy in recurrent spontaneous aborters: Analysis of anti-idiotypic antibodies directed against autologous T-cell receptors. Hum Reprod. 1999;14(3):650-5.

26- Beydoun H, Saftlas AF. Association of human leucocyte antigen sharing with recurrent spontaneous abortions. Tissue Antigens. 2005;65(2):123-35.

27- Takeshita T. Diagnosis and treatment of recurrent miscarriage associated with immunologic disorders: Is paternal lymphocyte immunization a relic of the past? J Nippon Med Sch. 2004;71(5):308-13.

28- Szpakowski A, Malinowski A, Głowacka E, Wilczyński JR, Kolasa D, Dyński M, et al. The influence of paternal lymphocyte immunization on the balance of Th1/Th2 type reactivity in women with unexplained recurrent spontaneous abortion. Ginekol Pol. 2000;71(6):586-92. [Polish]

29- Wu L, Luo LH, Zhang YX, Li Q, Xu B, Zhou GX, et al. Alteration of Th17 and Treg cells in patients with unexplained recurrent spontaneous abortion before and after lymphocyte immunization therapy. Reprod Biol Endocrinol. 2014;12:74.

30- Zare A, Saremi A, Hajhashemi M, Kardar GA, Moazzeni SM, Pourpak Z, et al. Correlation between serum zinc levels and successful immunotherapy in recurrent spontaneous abortion patients. J Hum Reprod Sci. 2013;6(2):147-51.

31- Saini V, Arora S, Yadav A, Bhattacharjee J. Cytokines in recurrent pregnancy loss. Clin Chim Acta. 2011;412(910):702-8.

32- Gao L, Zhang JP, Chen H, Zhang SN, Chen LB, Tan JP, et al. Characteristics of immune cell changes before and after immunotherapy and their clinical significance in patients with unexplained recurrent spontaneous abortion. Genet Mol Res. 2014;13(1):1169-78.

33- Fu B, Li X, Sun R, Tong X, Ling B, Tian Z, et al. Natural killer cells promote immune tolerance by regulating inflammatory TH17 cells at the human maternal-fetal interface. Proc Natl Acad Sci U S A. 2013;110(3):E231-40. 34- Yokoo T, Takakuwa K, Ooki I, Kikuchi A, Tamura M, Tanaka K. Alteration of TH1 and TH2 cells by intracellular cytokine detection in patients with unexplained recurrent abortion before and after immunotherapy with the husband's mononuclear cells. Fertil Steril. 2006;85(5):1452-8.

35- Wang WJ, Hao CF, Qu QL, Wang X, Qiu LH, Lin QD. The deregulation of regulatory $T$ cells on interleukin-17producing $\mathrm{T}$ helper cells in patients with unexplained early recurrent miscarriage. Hum Reprod. 2010;25(10):2591-6.

36- Yang H, Qiu L, Chen G, Ye Z, Lü C, Lin Q. Proportional change of CD4+CD25+ regulatory $\mathrm{T}$ cells in decidua and peripheral blood in unexplained recurrent spontaneous abortion patients. Fertil Steril. 2008;89(3):656-61.

37- Lee SK, Kim JY, Hur SE, Kim CJ, Na BJ, Lee M, et al. An imbalance in interleukin-17-producing $\mathrm{T}$ and Foxp3+ regulatory $\mathrm{T}$ cells in women with idiopathic recurrent pregnancy loss. Hum Reprod. 2011;26(11):2964-71.

38- Kim DJ, Lee SK, Kim JY, Na BJ, Hur SE, Lee M, et al.

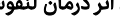

spontaneous abortion. Arch Gynecol Obstet. 2005;272(2):95-108.

5- Garrido-Gimenez C, Alijotas-Reig J. Recurrent miscarriage: Causes, evaluation and management. Postgrad Med J. 2015;91(1073):151-62.

6- Sugiura-Ogasawara M, Ozaki Y, Katano K, Suzumori N, Kitaori T, Mizutani E. Abnormal embryonic karyotype is the most frequent cause of recurrent miscarriage. Hum Reprod. 2012;27(8):2297-303.

7- Kwak-Kim J, Yang KM, Gilman-Sachs A. Recurrent pregnancy loss: A disease of inflammation and coagulation. J Obstet Gynaecol Res. 2009;35(4):609-22.

8- Mor G, Cardenas I, Abrahams V, Guller S. Inflammation and pregnancy: The role of the immune system at the implantation site. Ann N Y Acad Sci. 2011;1221(1):80-7.

9- Wegmann TG, Lin H, Guilbert L, Mosmann TR. Bidirectional cytokine interactions in the maternal-fetal relationship: Is successful pregnancy a TH2 phenomenon?. Immunol Today. 1993;14(7):353-6.

10- Bates MD, Quenby S, Takakuwa K, Johnson PM, Vince GS. Aberrant cytokine production by peripheral blood mononuclear cells in recurrent pregnancy loss? Hum Reprod. 2002;17(9):2439-44.

11- Saito S, Nakashima A, Shima T, Ito M. Th1/Th2/Th17 and regulatory $\mathrm{T}$-cell paradigm in pregnancy. Am $\mathrm{j}$ Reprod Immunol. 2010;63(6):601-10.

12- Dong C. TH17 cells in development: An updated view of their molecular identity and genetic programming. Nat Rev Immunol. 2008;8(5):337-48.

13- Ouyang W, Kolls JK, Zheng Y. The biological functions of $\mathrm{T}$ helper 17 cell effector cytokines in inflammation. Immunity. 2008;28(4):454-67.

14- Korn T, Bettelli E, Oukka M, Kuchroo VK. IL-17 and Th17 Cells. Annu Rev Immunol. 2009;27:485-517.

15- Heidt S, Segundo DS, Chadha R, Wood KJ. The impact of Th17 cells on transplant rejection and the induction of tolerance. Curr Opin Organ Transplant. 2010;15(4):45661.

16- Hanidziar D, Koulmanda M. Inflammation and the balance of Treg and Th17 cells in transplant rejection and tolerance. Curr Opin Organ Transplant. 2010;15(4):411-5.

17- Yoshida S, Haque A, Mizobuchi T, Iwata T, Chiyo M, Webb TJ, et al. Anti-type $\mathrm{V}$ collagen lymphocytes that express IL-17 and IL-23 induce rejection pathology in fresh and well-healed lung transplants. Am J Transplant. 2006;6(4):724-35.

18- Corthay A. How do regulatory T cells work?. Scand J Immunol. 2009;70(4):326-36.

19- Nakashima A, Shima T, Inada K, Ito M, Saito S. The balance of the immune system between $\mathrm{T}$ cells and NK cells in miscarriage. Am J Reprod Immunol. 2012;67(4):304-10.

20- Nakashima A, Ito M, Shima T, Bac ND, Hidaka T, Saito S. Accumulation of IL-17-positive cells in decidua of inevitable abortion cases. Am J Reprod Immunol. 2010;64(1):4-11.

21- Lee SK, Kim JY, Lee M, Gilman-Sachs A, Kwak-Kim J. Th17 and regulatory T cells in women with recurrent pregnancy loss. Am J Reprod Immunol. 2012;67(4):3118.

22- Fu B, Tian Z, Wei H. TH17 cells in human recurrent pregnancy loss and pre-eclampsia. Cell Mol Immunol. 2014;11(6):564-70.

23- Pandey MK, Agrawal S. Induction of MLR-Bf and protection of fetal loss: A current double blind randomized trial of paternal lymphocyte immunization 
before and after lymphocyte immunotherapy. Am J Reprod Immunol. 2012;68(2):164-74.

42- Liu YS, Wu L, Tong XH, Wu LM, He GP, Zhou GX, et al. Study on the relationship between Th17 cells and unexplained recurrent spontaneous abortion. Am J Reprod Immunol. 2011;65(5):503-11.

43- Messaoudi S, Al-Khateeb GM, Dendana M, Sater MS, Jazia KB, Nouira $M$, et al. Genetic variations in the interleukin-21 gene and the risk of recurrent idiopathic spontaneous miscarriage. Eur Cytokine Netw. 2011;22(2):123-6.

44- Saifi B, Rezaee SA, Tajik N, Ahmadpour ME, Ashrafi $\mathrm{M}$, Vakili R, et al. Th17 cells and related cytokines in unexplained recurrent spontaneous miscarriage at the implantation window. Reprod Biomed Online. 2014;29(4):481-9.
Intravenous immunoglobulin G modulates modulates periphera blood Th17 and Foxp3(+) regulatory T cells in pregnant women with recurrent pregnancy loss. Am J Reprod Immunol. 2014;71(5):441-50.

39- Bansal AS, Bajardeen B, Thum MY. The basis and value of currently used immunomodulatory therapies in recurrent miscarriage. J Reprod Immunol. 2012;93(1):41-51.

40- Gharesi Fard B, Zolghadri J, Kamali Sarvestani E. Effect of leukocyte therapy on tumor necrosis factoralpha and interferon-gamma production in patients with recurrent spontaneous abortion. Am J Reprod Immunol. 2008;59(3):242-50.

41- Liang P, Mo M, Li GG, Yin B, Cai J, Wu T, et al. Comprehensive analysis of peripheral blood lymphocytes in 76 women with recurrent miscarriage 